\title{
Castrum Novum
}

Chronique des campagnes de septembre 2010 et septembre 2011

Marie-Laurence Haack, Sara Nardi-Combescure, Grégoire Poccardi et Flavio Enei

AOROC (UMR 8546 CNRS-ENS), Université de Picardie Jules Verne, Université Lille 3 (HALMAIPEL - UMR 8164), Soprintendenza archeologica per i Beni Archeologici dell'Etruria meridionale et Museo civico di Santa Marinella (éd.)

\section{(2) OpenEdition Journals}

\section{Édition électronique}

URL : http://journals.openedition.org/cefr/616

DOI : $10.4000 /$ cefr.616

ISSN : 2282-5703

Éditeur

École française de Rome

Référence électronique

Marie-Laurence Haack, Sara Nardi-Combescure, Grégoire Poccardi et Flavio Enei, « Castrum Novum », Chronique des activités archéologiques de l'École française de Rome [En ligne], Italie centrale, mis en ligne le 19 décembre 2012, consulté le 14 novembre 2019. URL : http://journals.openedition.org/cefr/616 ; DOI : $10.4000 /$ cefr.616

Ce document a été généré automatiquement le 14 novembre 2019.

(c) École française de Rome 


\section{Castrum Novum}

Chronique des campagnes de septembre 2010 et septembre 2011

\section{Marie-Laurence Haack, Sara Nardi-Combescure, Grégoire Poccardi et} Flavio Enei

AOROC (UMR 8546 CNRS-ENS), Université de Picardie Jules Verne, Université Lille 3 (HALMAIPEL - UMR 8164), Soprintendenza archeologica per i Beni Archeologici dell'Etruria meridionale et Museo civico di Santa Marinella (éd.)

1 La mission a été créée à l'automne 2009 par une équipe pluri-universitaire et francoitalienne $^{1}$ dans le but d'étudier le site de Castrum Novum, colonie romaine maritime, dont la date de fondation remonte à la première moitié du III siècle av. J.-C. ${ }^{2}$. La colonie était située sur le territoire de l'ancienne cité étrusque de Caere (Cerveteri), à un emplacement qui correspond à l'actuelle localité de Santa Marinella (fig. 1). 
Fig. 1 - Castrum Novum. Localisation du site archéologique de Castrum Novum.

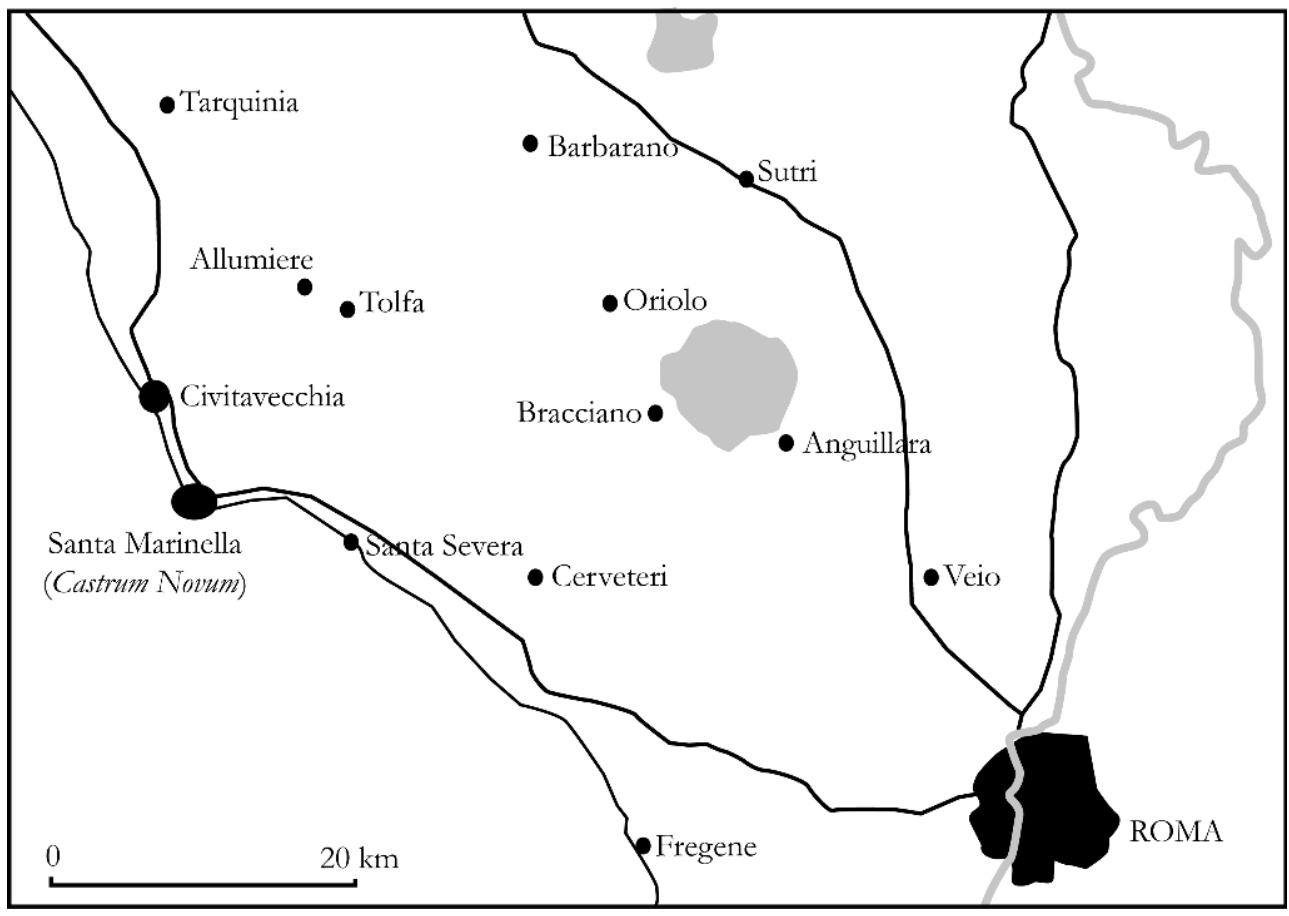

2 Actuellement, les restes de la ville antique se trouvent sur des terrains d'une superficie d'environ 3 hectares appartenant, pour une moitié, à la Soprintendenza per $i$ Beni Archeologici per l'Etruria Meridionale et, pour l'autre, à la famille Alibrandi. Le site a été identifié dans la seconde moitié du XVIII ${ }^{\mathrm{e}}$ siècle et plusieurs campagnes de fouilles ont alors été entreprises sous le pontificat de Pie VI, entre 1777 et 1779, puis par la Reverenda Camera Apostolica entre 1794 et 1795 ; dans les années 1970, la Soprintendenza per i Beni Archeologici dell'Etruria Meridionale a mené des fouilles limitées au nord du site.

Dans le cadre de cette nouvelle mission, deux campagnes d'exploration se sont déroulées en septembre 2010 et septembre 2011 au cours desquelles les activités de terrain ont consisté à nettoyer et à relever un balneum dit « Le Guardiole » (secteur II de la zone A) et les vestiges conservés en bord de mer (en particulier un autre balneum), visibles en section, sur la portion de la côte située entre " Torre Chiaruccia » et "Casale Alibrandi » (secteurs I et II de la zone B). Une prospection sous-marine a également été entreprise par la société Poséidon sous la responsabilité d'Ed. Bruni et de St. Giorgi dans la partie immergée de la ville (zone $\mathrm{C}$ ) et une prospection terrestre géophysique par l'équipe de l'Université de Sienne dirigée par S. Campana et F. Pericci (fig. 2). 
Fig. 2 - Castrum Novum. Cliché aérien du site de Castrum Novum.

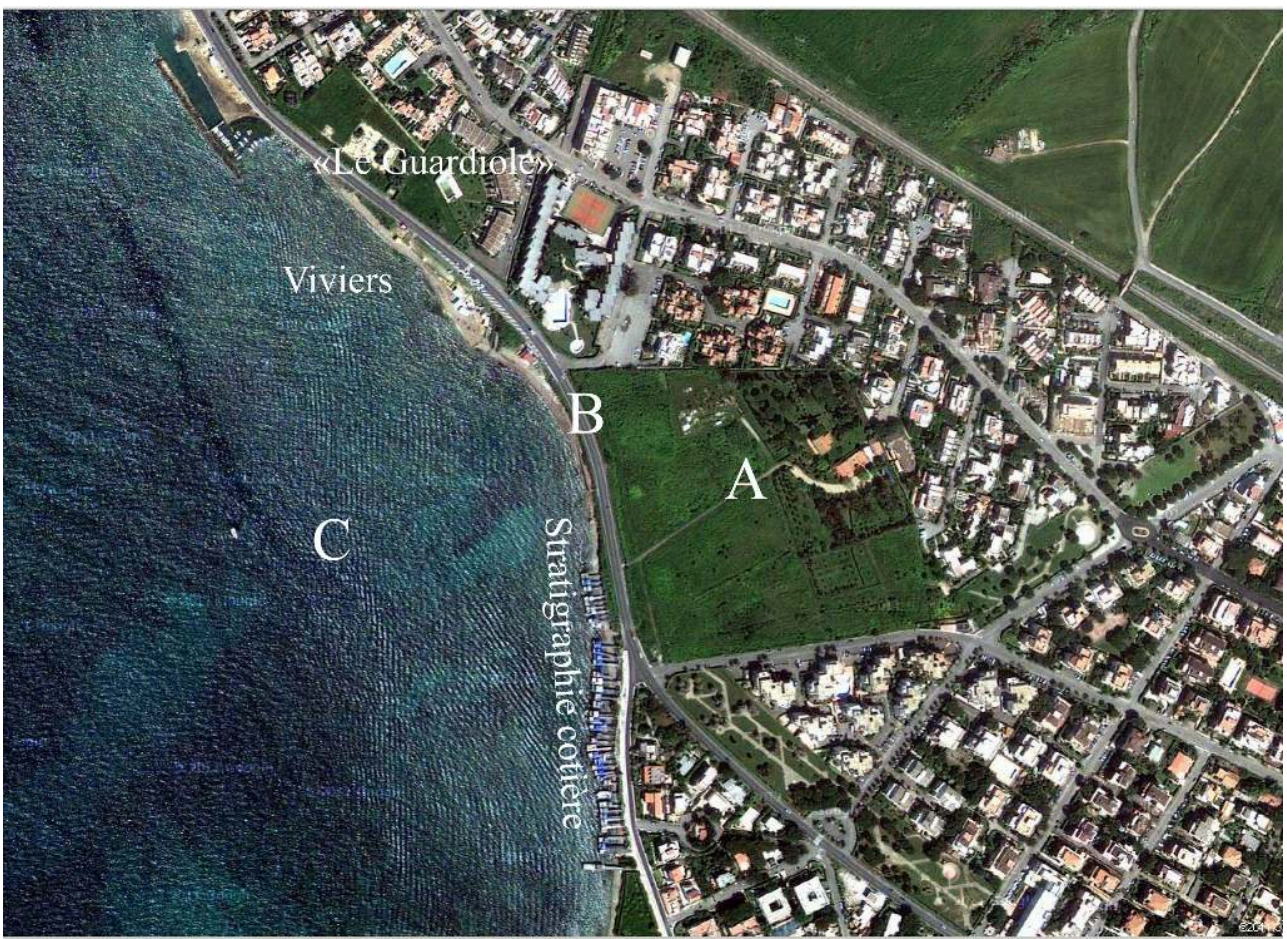

\section{Zone A : Le complexe balnéaire de "Le Guardiole » (responsable S. Nardi Combescure)}

4 Il s'agit d'un établissement de bains partiellement dégagé lors d'une campagne de fouilles conduite au début des années 1970 par la Soprintendenza per $i$ Beni Archeologici per l'Etruria Meridionale (fig. 3 et 4).

Fig. 3 - Castrum Novum. Plan du balneum de « Le guardiole » (zone A, secteur 1).
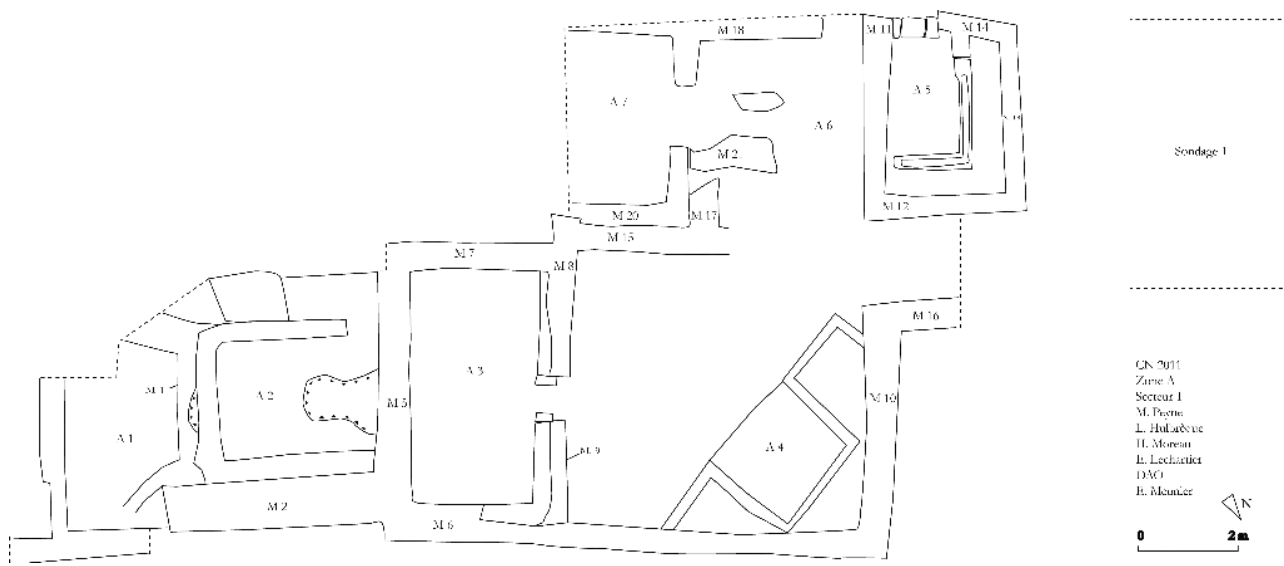
Fig. 4 - Castrum Novum. Vue générale du balneum de « le Guardiole » (zone A, secteur 1).

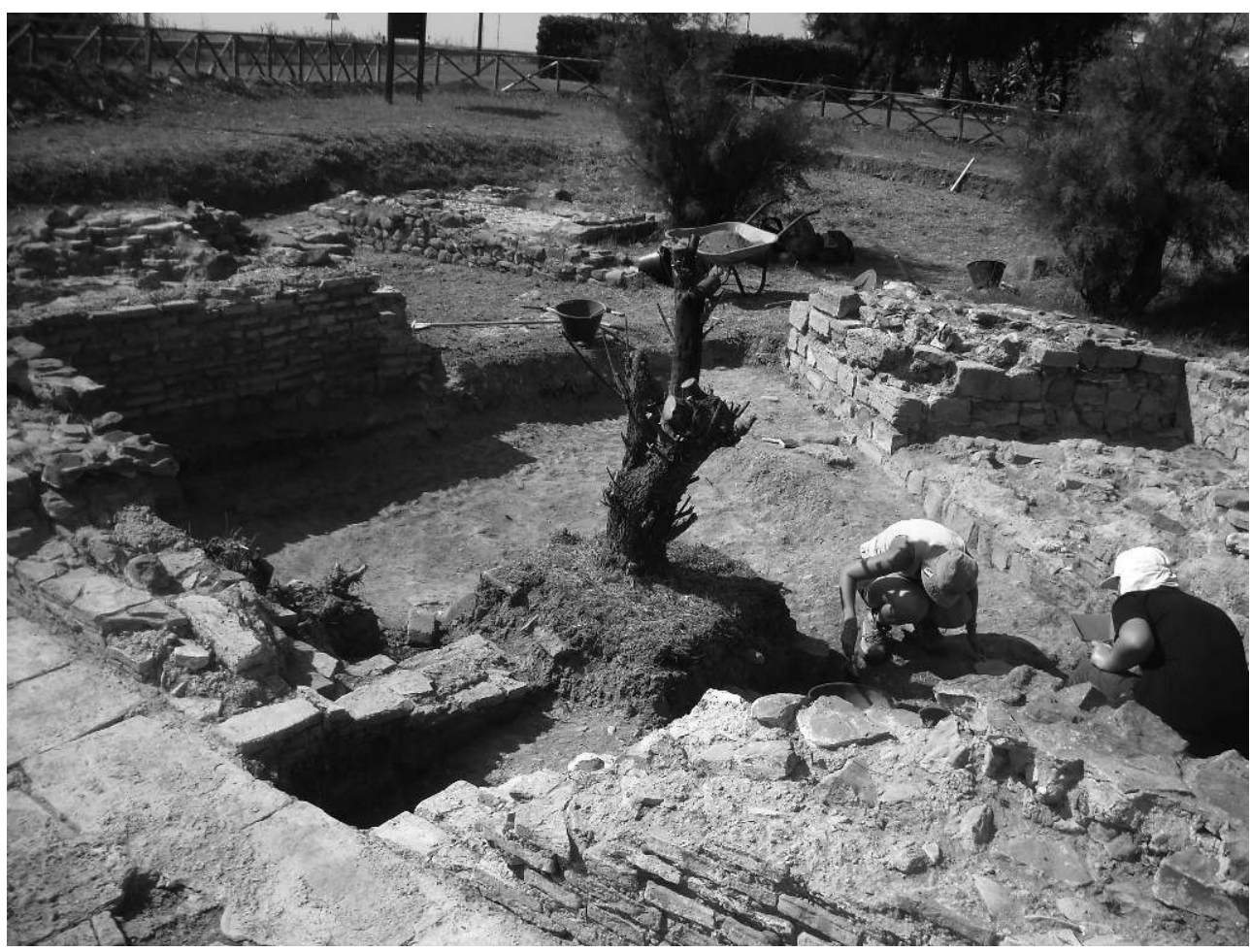

5 Cet établissement est situé sur le côté Ouest de la via Aurelia vetus (abandonnée au début de l'époque antonine) au Nord du site de Castrum Novum.

Ce balneum se trouve aussi à $50 \mathrm{~m}$ environ au Sud d'un autre édifice qui a été identifié comme les restes d'une villa romaine, située de l'autre côté de la via Aurelia vetus et donc sans aucun lien entre eux. La mission de septembre 2010 a d'abord été consacrée au nettoyage de ce balneum, car celui-ci était entièrement couvert de végétation et les restes visibles étaient en mauvais état de conservation.

7 Dans l'état actuel de nos connaissances, le balneum se compose de sept pièces. La pièce A1, qui n'est plus visible actuellement, conserve les traces d'une mosaïque noire et blanche. La pièce A2 abrite un bassin recouvert d'un mortier hydraulique alimenté par un canal situé au Sud. La vasque semble être placée sur une structure plus ancienne, coupée par un mur et par un canal. La pièce A3 est un espace chauffé, puisqu'au moment des fouilles de 1970, cet espace était doté d'un hypocauste ; les parois internes des murs sont composées d'assises régulières de briques et de tuiles, disposées en alternance. La pièce A4 semble remonter à une phase postérieure à l'abandon du complexe. Les fondations de ces structures sont conservées, mais il n'y a pas de trace du pavement qui devait être constitué par une épaisse strate de terre battue.

8 À proximité, on trouve de petites latrines (A5), encore en bon état de conservation avec un parement en opus spicatum et entourées sur deux côtés par un petit canal recouvert d'un mortier hydraulique. À un niveau inférieur, sont situées deux autres pièces qui, à l'origine, communiquaient.

9 L'accès au praefurnium (A6) s'ouvre sur un couloir de service qui fera l'objet d'une fouille en 2012. Comme dans le cas de la pièce A3, la pièce A7 est dotée d'un système de chauffage par hypocauste, avec un sol en tuiles et des parois internes faites d'assises régulières de briques et de tuiles. 
10 Le matériel céramique retrouvé, qui n'a pas encore entièrement été étudié, semble à première vue dater d'entre le début du $\mathrm{I}^{\mathrm{er}}$ siècle et la fin du $\mathrm{II}^{\mathrm{e}}$ siècle après $\mathrm{J}$. -C.

\section{Zone B : la stratigraphie de la zone littorale (responsable G. Poccardi)}

11 La ligne de côte se caractérise par une coupe stratigraphique naturelle de plusieurs centaines de mètres. Cette stratigraphie a été divisée en deux secteurs distincts : le premier, au Sud, se caractérise par des niveaux d'occupation de l'Âge du Fer ; le second, au Nord, abrite toute une série de structures appartenant à l'agglomération romaine (fig. 5).

Fig. 5 - Castrum Novum. Cliché aérien du secteur 2 de la zone B.

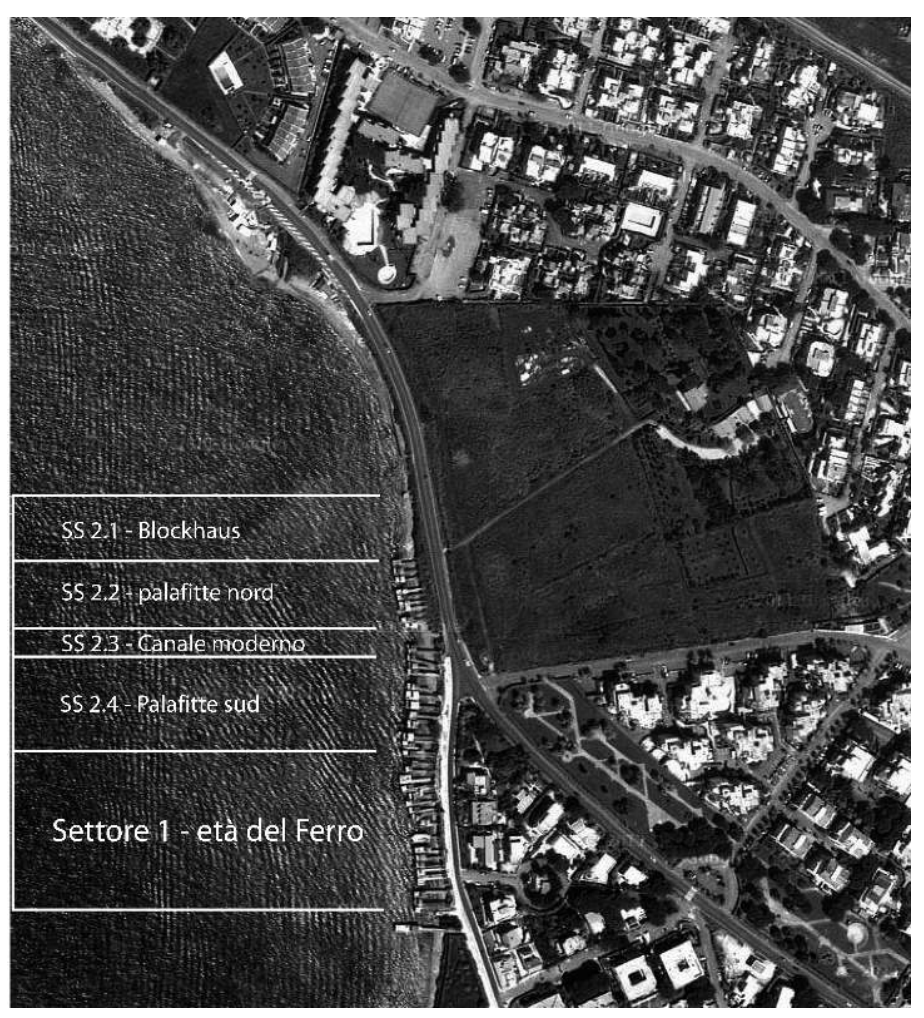

\section{La campagne de 2010}

Lors de la campagne de septembre 2010, l'étude s'est limitée à l'analyse d'une structure monumentale identifiée comme un balneum et à trois tombes tardives qui avaient fait l'objet de fouilles clandestines, peu de temps auparavant, situées à l'extrémité sud du secteur 2. Ce bain est matérialisé par une structure en abside dont dépendait un couloir de service (fig. 6). 
Fig. 6 - Castrum Novum. Vue de l'abside et du couloir de service du balneum situé dans le soussecteur 2.4 - Palafitte sud.

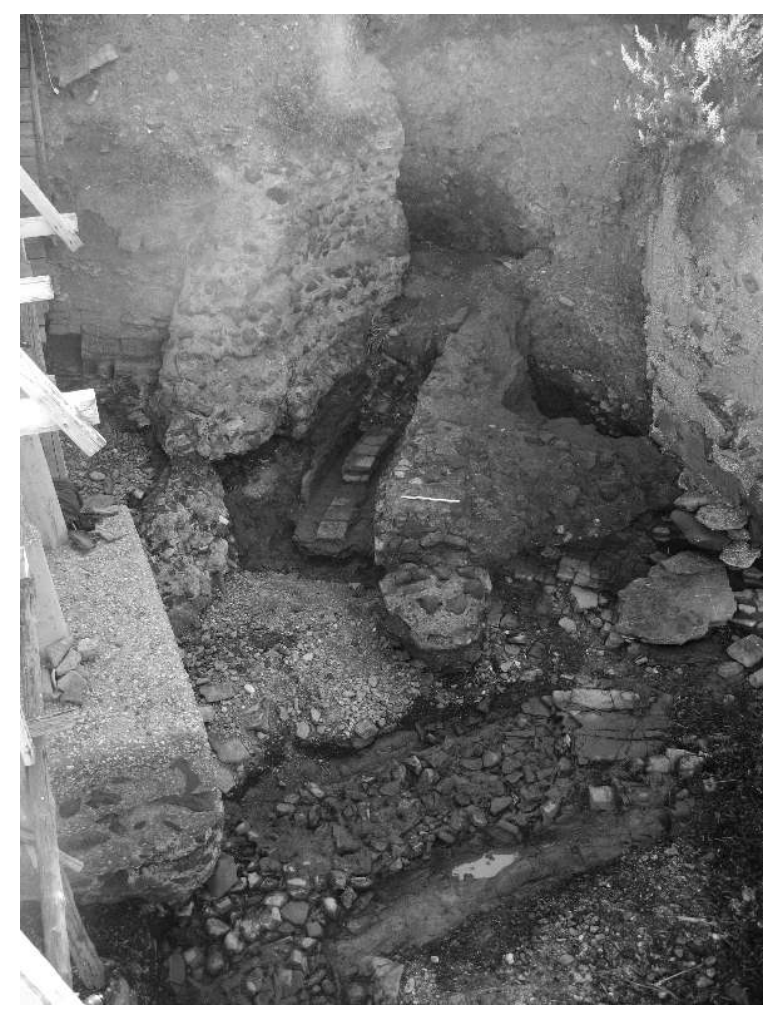

13 L'emploi de l'opus reticulatum mixtum à chaînages de briques permet de dater cette structure de 100 après J.-C. environ. À l'intérieur de l'abside, conservée sur une hauteur d'environ $1,50 \mathrm{~m}$, on aperçoit une ouverture horizontale de forme rectangulaire avec une marque circulaire qui pourrait faire penser à une testudo alvei, c'est-à-dire à une plaque de métal servant à transmettre rapidement la chaleur du foyer à un éventuel bassin.

Le couloir de service, d'une largeur de 2,5 pieds romains $(0,75 \mathrm{~m})$, a été en partie dégagé $\mathrm{du}$ sable et des galets qui le recouvraient. Cela a permis la mise au jour du niveau de circulation ainsi que d'un canal d'évacuation des restes de cendres d'un four et peutêtre des eaux usées de la baignoire. Enfin, ont été retrouvés les restes d'un couloir rectiligne situé au même niveau que le couloir de service de l'abside. Des traces du bain sont encore visibles en coupe, plus au Sud, sur 7,40 m (fig. 7). 
Fig. 7 - Castrum Novum. Vue en stratigraphie des structures du balneum situé dans le Sous-secteur 2.4 - Palafitte sud.

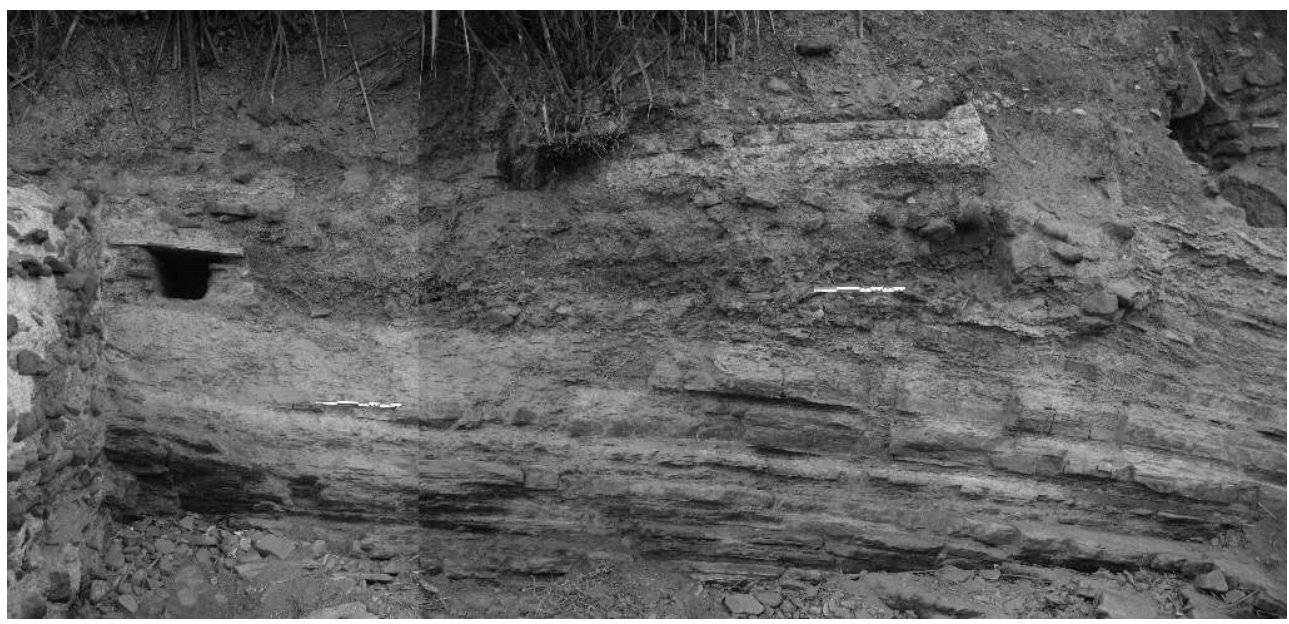

La stratigraphie permet de voir les restes d'une vasque en mortier friable noir, recouvert d'un mortier hydraulique rosé, qui recouvre un canal à la fonction imprécise, ainsi, qu'au Sud, les restes d'un égout. En continuant vers le Sud, on distingue la série de trois tombes tardives à bâtière.

\section{La campagne de $2011^{3}$}

Grâce à l'expérience acquise lors de la première campagne, il a été décidé de lancer, pour l'année 2011, l'étude complète de la stratigraphie côtière en commençant à partir du nord, au niveau des terrains libres de construction, là où aurait été situé le cœur de la colonie romaine. Pour des raisons de temps, la portion à étudier englobait seulement deux des quatre sous-sections du secteur 2 (1-Blockhaus et 2-Palafitte nord) représentant $90 \mathrm{~m}$ de stratigraphie environ.

Le travail a consisté à réaliser les relevés stratigraphiques et parfois planimétriques des structures visibles.

Pour les relevés stratigraphiques de la paroi, il s'est agi, non pas de réaliser un unique relevé de l'ensemble des $90 \mathrm{~m}$ de stratigraphie, mais de faire une série de relevés des structures visibles (murs, sols, égouts et autres) en prenant les cotes en altitude et par rapport à deux axes pré-établis pour les replacer in fine en plan et en section sur un document unique. Comme certaines structures émergent de la paroi, il a été procédé à leur relevé planimétrique (structure 19). Enfin, une couverture photographique de la paroi a été réalisée grâce à une série de clichés couvrant l'ensemble de la stratigraphie à étudier.

19 Un inventaire des structures visibles a été réalisé, mais il ne peut être considéré aujourd'hui comme définitif, en particulier pour la numérotation des vestiges.

Pour la sous Section 1 - Blockhaus, il est possible de voir :

21 - Structure 1: canal maçonné de 0,15 $\mathrm{m}$ de large sur 0,24 $\mathrm{m}$ de haut en petits blocs de pierre que l'on peut identifier comme un égout $\left(n^{\circ} 1\right)$ d'époque républicaine.

22 - Ensemble 2: niveau sans structure maçonnée, qui, à première vue, peut être interprété comme un niveau de circulation ne dépendant pas d'un édifice. 

base. Ces murs délimitent deux espaces dotés de sols en partie conservés en mosaïque noire et blanche.

24 - Structure 4 : égout $\left(\mathrm{n}^{\circ} 2\right)$ d'époque impériale, en partie saccagé durant l'hiver 2011. Il est composé d'un canal de 0,44 $\mathrm{m}$ de large pour $0,48 \mathrm{~m}$ de haut, construit de tuiles et de briques surmontées par deux tuiles posées en bâtière. Parmi les fragments tombés au sol, a été retrouvé un morceau de timbre épigraphique sur une brique en cours d'étude (fig. 8) .

Fig. 8 - Castrum Novum. Photographie de l'égout n 2 dans le sous-secteur 2.1 - Blockhaus.

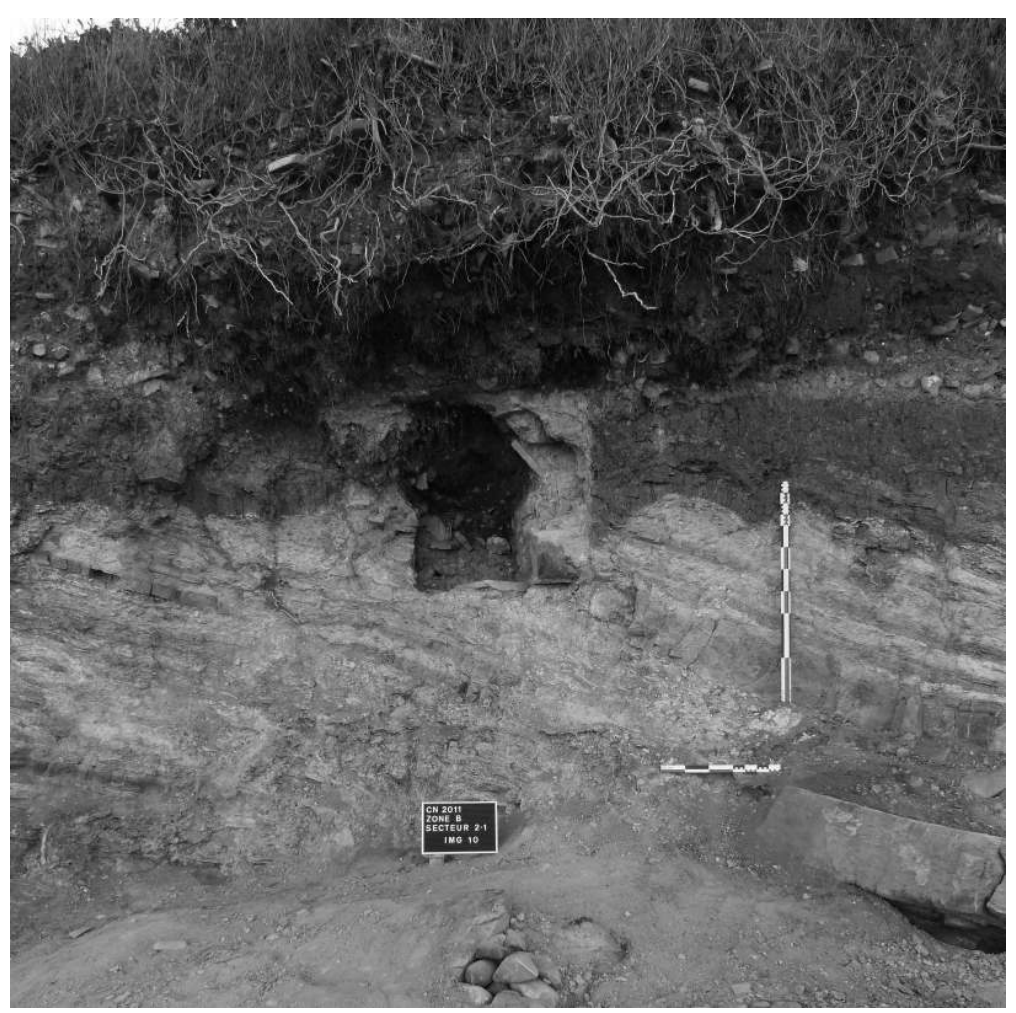

- Structure 5: égout $\left(\mathrm{n}^{\circ} 3\right)$ d'époque républicaine maçonné en blocs de pierre. L'ouverture de son canal est de 0,44 $\mathrm{m}$ de large et de 0,48 $\mathrm{m}$ de haut. Sa particularité par rapport aux autres vestiges est sa direction différente à environ 60 par rapport aux autres structures.

26 - Ensemble 6 : élément de mur avec sa fondation plus large sur lequel sont conservés des restes d'enduit à la base des deux parois et des fragments du sol de mosaïque à tesselles blanches au sud.

Pour la sous-section 2 - Palafitte nord :

28

: il s'agit de deux sous-ensembles superposés. Le premier est constitué de deux murs d'un bâtiment arasé, dont on ne voit que le niveau des fondations. À 0,25 m au-dessus, deux autres murs d'un édifice postérieur sont visibles, décalés vers le sud. Les niveaux de sol de l'édifice le plus récent ont été en partie conservés avec des restes de mosaïques (fig. 9).

Chronique des activités archéologiques de l'École française de Rome , Italie centrale 
Fig. 9 - Castrum Novum. Vue de la coupe stratigraphique au niveau des structures de l'ensemble $n^{\circ} 7$ et de l'égout d'époque impériale $n^{\circ} 16$ dans le sous-secteur 2.2 - Palafitte nord.

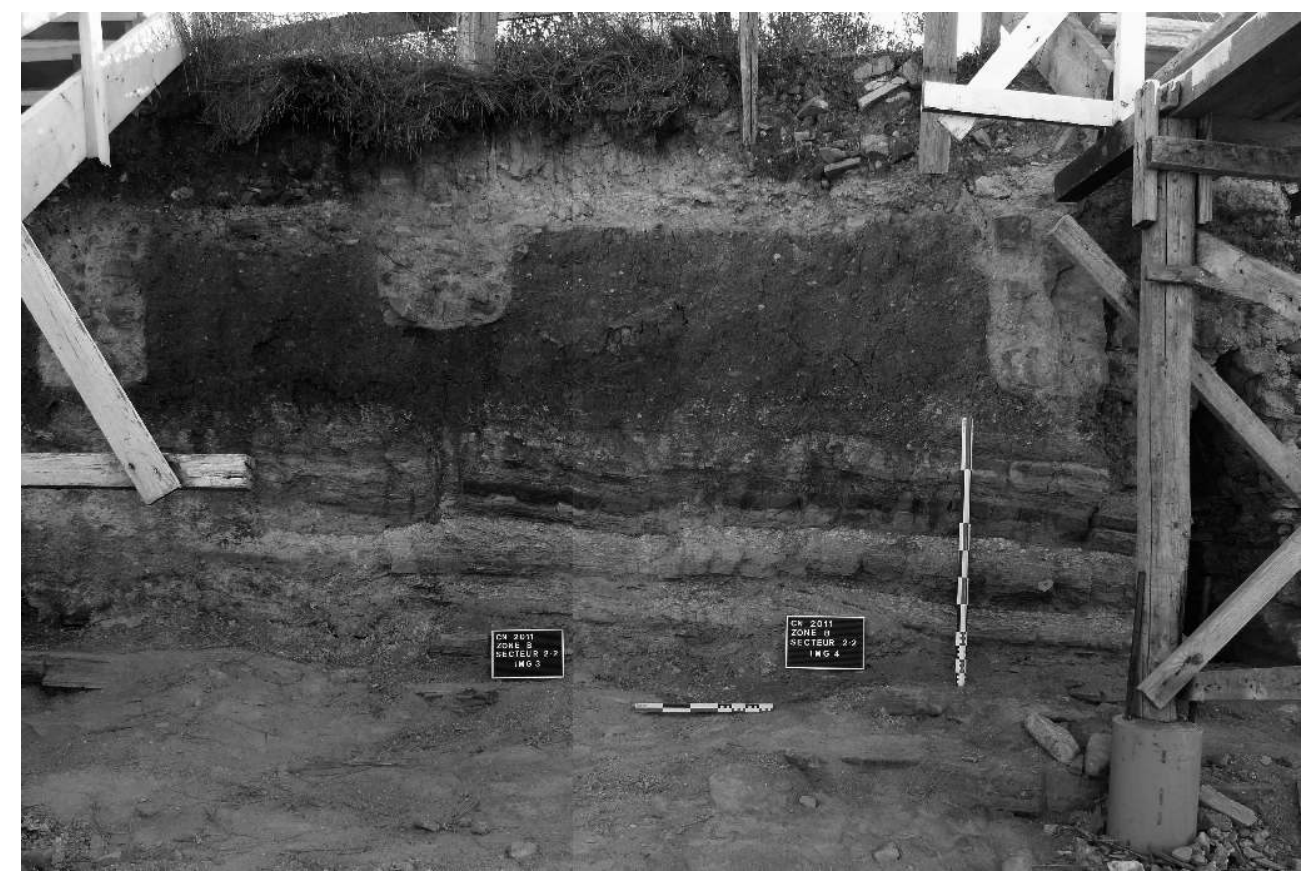

- Ensemble (8 et 9): deux murs accolés, un premier massif d'1,52 m d'épaisseur fortement arasé et un second de seulement 0,46 $\mathrm{m}$ d'épaisseur.

30 - Structure 10 : fondation de mur fort épaisse de 1,64 m.

31 - Structure 11 : fondation de mur, située au sud de la structure 10, d'épaisseur de 0,62 m au maximum.

32 - Structures 12 et $13:$ mur isolé arasé de $0,48 \mathrm{~m}$ de large, dont on voit principalement la fondation qui, elle, fait $0,58 \mathrm{~m}$ de large avec des traces d'un sol contre la paroi méridionale. Ce mur sert actuellement de soubassement à un pilier de la passerelle d'un cabanon moderne.

- Structure 14: élément de fondation sur lequel s'est appuyée la passerelle d'un cabanon.

34 - Ensemble 15: mur en opus reticulatum mixtum conservé sur près de 2,50 $\mathrm{m}$ de haut, fondation comprise. Des traces de l'enduit peint de couleur rouge sont visibles sur les parois (fig. 10). 
Fig. 10 - Castrum Novum. Mur $\mathrm{n}^{\circ} 15$ en opus reticulatum mixtum (avec sa fondation) dans la soussection 2 - Palafitte nord.

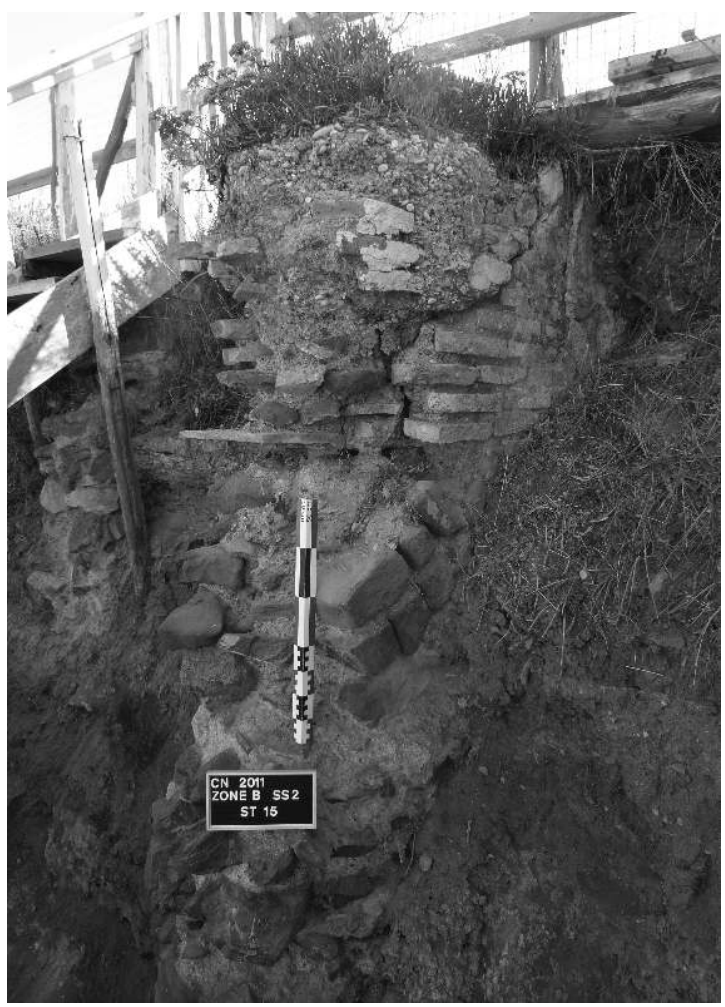

35 - Structure 16 : égout d'époque impériale maçonné en briques et couvert en bâtière de deux briques (bipedales). Il comporte un canal, d'une largeur de $0,45 \mathrm{~m}$ pour une hauteur de 1,36 m, que l'on peut suivre sur plus de $9 \mathrm{~m}$. Il se situe sous le niveau de sol d'un espace qui dépend de l'ensemble 7.

- Structure 17 (a-b): mur en opus reticulatum, axé nord-sud et conservé en deux tronçons sur une longueur de près de $6 \mathrm{~m}$. C'est sur ce mur que s'appuie la structure hydraulique 19.

37 - Structure 18 : noyau en opus caementicium du mur 17 en opus reticulatum, le parement ayant probablement été taillé, puisqu'une partie du mur a disparu.

38 - Ensemble 19: structure hydraulique en opus caementicium dont sont conservés un puits et un bassin au nord. Cette structure est accolée au mur 17b qui lui a servi d'appui (fig. 11). 
Fig. 11 - Castrum Novum. Cliché vertical et relevé du puits de la structure hydraulique $n^{\circ} 19$ qui s'appuie sur la section (b) du mur $\mathrm{n}^{\circ} 17$ en opus reticulatum.

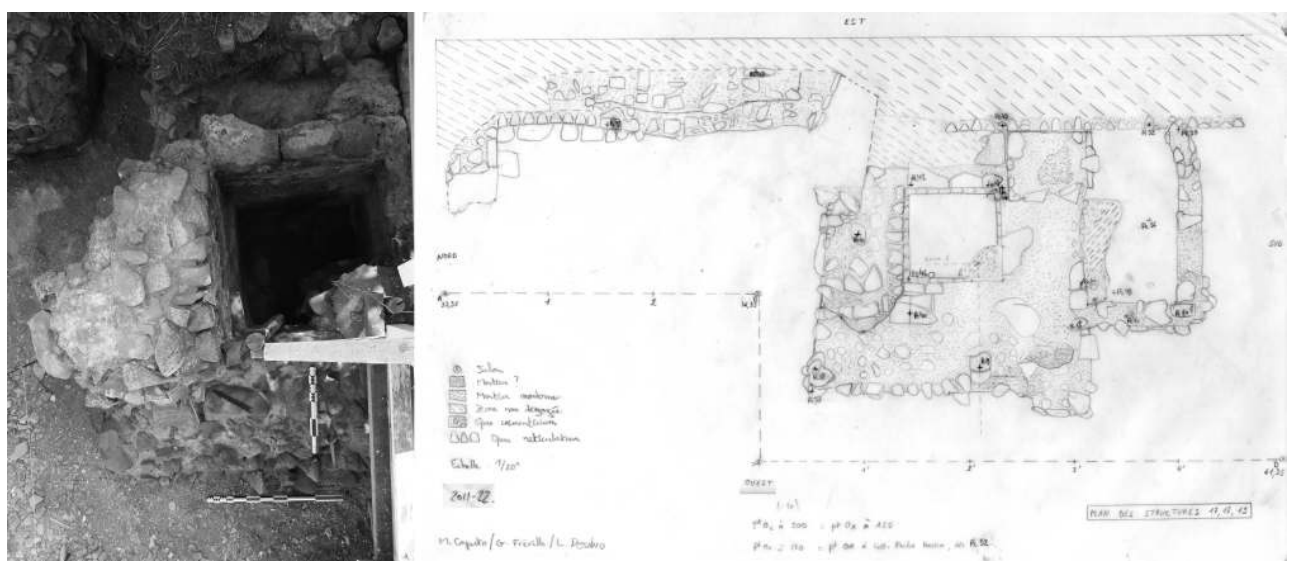
de Lille 3 Charles de Gaulle, une équipe de la société Poséidon menée par Ed. Bruni et par St. Giorgi a entrepris une prospection des fonds sous-marins à proximité du littoral de Castrum Novum grâce à l'utilisation d'un sonar installé sur un bateau. Les premières analyses ont permis de confirmer les résultats des explorations sous-marines antérieures et de commencer à dresser une carte précise des fonds au niveau de la ville antique. L'équipe a aussi repris l'étude des viviers antiques du bord de mer, certains étant encore visibles à quelques mètres du rivage. de l'Université de Sienne, sous la direction de S. Campana et de F. Pericci, grâce à un financement de l'UMR 8546 du CNRS - ENS Paris (AOROC). La zone prospectée à l'aide d'un magnétomètre couvrait une superficie d'un peu moins de 2 hectares sur la partie nord des terrains libres de constructions, appartenant à la Surintendance (fig. 9). Les 
premiers résultats montrent d'importantes structures au nord de la zone. Il est prévu de compléter l'enquête par la prospection de la partie méridionale du terrain appartenant à la famille Alibrandi.

\section{BIBLIOGRAPHIE}

V. Annovazzi, Castrum Novum, dans Notizie degli scavi di antichità, 1879, p. 136.

G. M. De Rossi, P. Di Domenico, L. Quilici, La via Aurelia da Roma a Forum Aureli, Rome, 1968

(Quaderni dell'Istitituto di topografia antica, 4).

S. Bastianelli, Centumcellae-Castrum Novum, Rome, 1944 (Italia romana : municipi e colonie, I, XIV).

Fl. Enei, Progetto Ager Caeretanus. Il littorale di Alsium : ricognizioni archeologiche nel territorio dei comuni di Ladispoli, Cerveteri e Fiumicino (Alsium, Caere, Ad Turres, Ceri), Ladispoli, 2001.

Enei et alii 2011 = Fl. Enei, M. L. Haack, S. Nardi Combescure, G. Poccardi, Castrum Novum. Storia e archeologia di una colonia romana nel territorio di Santa Marinella, Santa Marinella, 2011 (Quaderno, 1).

V. Fiocchi Nicolai, I cimiteri paleocristiani del Lazio. 1. Etruria Meridionale, Cité du Vatican, 1988.

P. A. Gianfrotta, Castrum Novum, Forma Italiae, Regio VII, III, Rome, 1972.

S. Nardi Combescure, Paesaggi d'Etruria Meridionale. L'entroterra di Civitavecchia dal II al XV secolo d.

C., Florence, 2002.

\section{NOTES}

1. La mission est financée par les centres de recherche, Halma-Ipel (UMR 8164, CNRS, Lille 3 et MCC) de l'Université de Lille 3 Charles de Gaulle, TRAME (EA 4284) et CRA (EA 4291) de l'Université de Picardie Jules Verne et AOROC (UMR 8546 de CNRS - ENS Paris), et bénéficie de l'aide matérielle du Museo del mare e della navigazione antica de Santa Severa.

2. Pour les problèmes de datation de la déduction de la colonie, Enei et alii 2011, p. 4 et p. 37.

3. Pour la campagne 2011, l'équipe qui a travaillé sur la stratigraphie de la zone B était composée de Matthieu Peyne (Université de Limoges), de Gontran Freville (Université de Lille 3), de Maxence Caputo (Université de Lille 3) et de Luca Desibio (Université de Roma3), avec l'assistance d'Elsa Perault (ENS Paris).

4. Des fragments de briques, pour la plupart pourvus de timbres anépigraphes, ont été récupérés par S. Giorgi. 
INDEX

Index géographique : Castrum Novum, Santa Marinella

Mots-clés : colonie romaine maritime, balneum, Étrurie, Caere, Cerveteri, Santa Marinella institutions AOROC (UMR 8546 CNRS-ENS), Université de Picardie Jules Verne, Soprintendenza archeologica per i Beni Archeologici dell'Etruria meridionale, Université de Lille 3 (UMR 8164 du CNRS HALMA-IPEL)

\section{AUTEURS}

\section{MARIE-LAURENCE HAACK}

AOROC (UMR 8546 CNRS-ENS) - haackml[at]yahoo.fr

\section{SARA NARDI-COMBESCURE}

Université de Picardie Jules Verne, AOROC (UMR 8546 CNRS-ENS) - saracombescure[at]free.fr GRÉGOIRE POCCARDI

Université Lille 3 (HALMA-IPEL - UMR 8164) - gregoire.poccardi[at]univ-lille3.fr

\section{FLAVIO ENEI}

Directeur, Museo civico di Santa Marinella 